\title{
Rotation Estimation for Wide-Angle Inverse Synthetic Aperture Radar Imaging
}

\author{
Wei Zhou, ${ }^{1}$ Chun-mao Yeh, ${ }^{2}$ Rui-jin Jin, ${ }^{1}$ Jian Yang, ${ }^{1}$ and Jian-she Song ${ }^{3}$ \\ ${ }^{1}$ Department of Electronic Engineering, Tsinghua University, Beijing 100084, China \\ ${ }^{2}$ Beijing Institute of Radio Measurement, Beijing 100854, China \\ ${ }^{3}$ Xian Research Institute of Hi-Technology, Xian 710025, China
}

Correspondence should be addressed to Wei Zhou; zhouw06@mails.thu.edu.cn

Received 19 July 2015; Revised 11 September 2015; Accepted 21 September 2015

Academic Editor: Sher Bahadar Khan

Copyright (C) 2016 Wei Zhou et al. This is an open access article distributed under the Creative Commons Attribution License, which permits unrestricted use, distribution, and reproduction in any medium, provided the original work is properly cited.

\begin{abstract}
To present focused ISAR imaging results in the homogenous range and cross-range domain, an integrated scheme is proposed to estimate both the targets equivalent rotational velocity (RV) and rotational center (RC). The RV estimation is improved by radial projection combined with keystone processing, and then the RC is estimated through image entropy minimization. Finally, delicate imaging results may be obtained for wide-angle scenarios. Experiment results are provided to demonstrate the effectiveness of the proposed method.
\end{abstract}

\section{Introduction}

Inverse synthetic aperture radar (ISAR) may provide highresolution images for noncooperative moving targets [1], and it has been widely used for both military and civilian purposes $[2,3]$. ISAR gets high range resolution by transmitting and processing waveform with large bandwidth and gets high cross-range resolution by coherently processing echoes from different aspect angles. Generally speaking, with careful imaging interval selection $[4,5]$, the target's movement is usually considered as planar rotation relative to the radar after effective translational motion compensation (TMC).

When the integrated aspect angle interval is small, ISAR images may be formed using the efficient range-Doppler (RD) algorithm. For better image understanding and target feature extraction, it is more preferable to rescale the $\mathrm{RD}$ image into the homogeneous range-cross-range domain. However, the cross-range scaling factor (SF) is related to the rotational velocity (RV) of the target, which is usually unknown, and it should be estimated for a noncooperative moving target. Furthermore, when echoes are collected from a relatively large aspect angle interval, more sophisticated imaging algorithms such as convolution back projection
(CBP) algorithm or the polar format algorithm (PFA) should be used to compensate the scattering centers' migration through resolution cells (MTRC). In these cases, both the RV and the equivalent rotational center (RC) should be known, which are necessary for high-resolution ISAR image forming and understanding.

In recent years, a number of methods have been proposed for the RV estimation from collected wideband echoes. These data-driven methods may be roughly categorized into three classes. The first is to search the RV which provides the best image focusing quality using the PFA [6], CBP [7], or a frame processing [8] structure. These methods are limited by the computation burden, and their effectiveness may be undermined by the unknown RC. The second is to get the RV by exploiting the high-order phase coefficients [9-15] of prominent scattering centers. This kind of methods may be limited by the scattering centers' MTRC as well as the computational burden. The third is to search the RV by exploiting the target's pose difference on RD image series [16-18]. However, the extraction and association of scattering centers between neighboring images may be difficult to implement due to the aspect angle change. In order to avoid the extraction and association of scattering centers, a method based on image 
rotation correlation was proposed in [17], but the accuracy may also be undermined by the unknown RC. Later, an equivalent algorithm using two-dimensional (2D) Fourier transform (FT) and polar mapping was proposed in [18] to eliminate the impact of the target's unknown RC. However, the computation is still not so efficient for the $2 \mathrm{D}$ interpolation in each iteration.

In this paper, a method combining the 2D FT with radial projection is proposed to estimate the RV. The radial projection is used to convert the $2 \mathrm{D}$ maximum correlation into a one-dimensional (1D) polar curve matching problem. Then, a representation of the polar curves using the cosine series is introduced to avoid the interpolation operation. With an easy initialization, the 1D maximization problem can be solved in a few iterations more efficiently.

There have been few reports about the estimation of the target's RC in open literatures. Here, on the basis of effective RV estimation, the RC may be found with the minimum entropy criterion. With all the rotational parameters, the delicate CBP imaging algorithm is then used to generate sizescaled ISAR images with high resolution.

The paper is organized as follows. The existing RV estimation methods are introduced in Section 2 based on image correlation and polar mapping. Then, the improved $\mathrm{RV}$ estimation method is proposed in Section 3 based on radial projection. With the known $\mathrm{RV}$, the equivalent $\mathrm{RC}$ of ISAR targets is determined by a minimum entropy criterion. Finally, experimental results with both simulated and collected wideband echoes are provided to demonstrate the effectiveness of the proposed parameter estimation scheme.

\section{RV Estimation Based on Image Correlation}

After careful imaging interval selection and effective TMC, an ISAR target may be considered as a uniformly planar rotating object as shown in Figure 1. $\left(r_{o}, \theta_{o}\right)$ is the polar coordinates for an arbitrary scattering center $P\left(x_{o}, y_{o}\right), r_{a}$ is the distance from the radar to the RC, and the far-field condition is satisfied when $r_{a} \gg r_{o}$. For a RD image formed around $t_{m}$, the scatter center is mapped on the discrete image domain as [17]

$$
\begin{aligned}
& X\left(t_{m}\right)=\frac{\left[r_{c}+x_{o} \cos \left(\omega_{o} t_{m}\right)-y_{o} \sin \left(\omega_{o} t_{m}\right)\right]}{\eta_{r}}, \\
& Y\left(t_{m}\right)=\frac{\left[x_{o} \sin \left(\omega_{o} t_{m}\right)+y_{o} \cos \left(\omega_{o} t_{m}\right)\right]}{\eta_{a}},
\end{aligned}
$$

where $\omega_{o}$ is the unknown RV, $r_{c}$ is the range bias of RC and is supposed to be a constant after TMC, $\eta_{r}=c /\left(2 f_{s}\right)$ is the range SF, $\eta_{a}=\lambda f_{r} /\left(2 M \omega_{o}\right)$ is cross-range SF, $f_{s}$ is the sampling frequency, $c$ is the velocity of light, $\lambda$ is the wavelength, $f_{r}$ is the pulse repetition frequency, and $M$ is the number of accumulated pulses for this RD image.

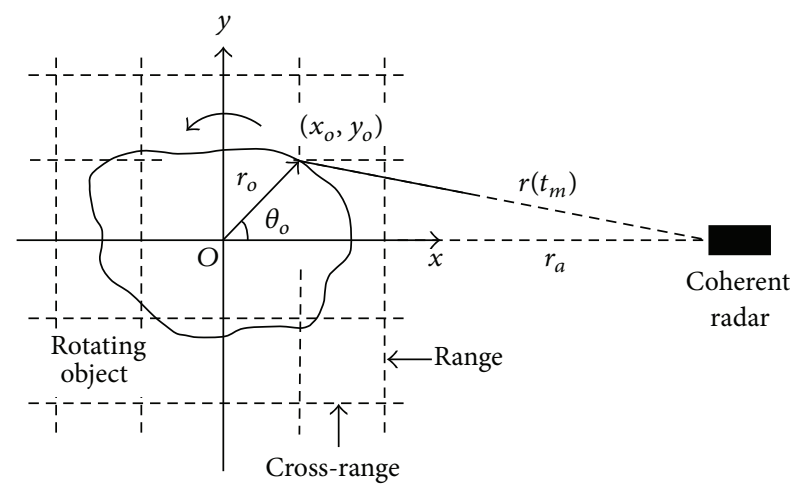

FIGURE 1: Rotating target imaging geometry.

Suppose that two RD images are formed at $t_{m 1}$ and $t_{m 2}$ by equally dividing all received echoes in the coherent processing interval (CPI). Then, the scatters' coordinates on two $\mathrm{RD}$ images are related as follows:

$$
\left[\begin{array}{l}
X\left(t_{m 2}\right) \\
Y\left(t_{m 2}\right)
\end{array}\right]=H\left(\theta_{d}\right)\left[\begin{array}{l}
X\left(t_{m 1}\right) \\
Y\left(t_{m 1}\right)
\end{array}\right]+\left[\begin{array}{l}
X_{c} \\
Y_{c}
\end{array}\right] .
$$

There is a displacement term $\left[X_{c}, Y_{c}\right]^{T}$ in (2) caused by the range bias of RC, $\theta_{d}=\omega_{o}\left(t_{m 2}-t_{m 1}\right)$ is the aspect angle difference between the neighboring RD images, and $H\left(\theta_{d}\right)$ is a generalized rotational matrix, given by

$$
\begin{aligned}
H\left(\theta_{d}\right) & =\left[\begin{array}{ll}
h_{11} & h_{12} \\
h_{21} & h_{22}
\end{array}\right] \\
& =\left[\begin{array}{cc}
\cos \left(\theta_{d}\right) & -\left(\frac{\eta_{a}}{\eta_{r}}\right) \sin \left(\theta_{d}\right) \\
\left(\frac{\eta_{r}}{\eta_{a}}\right) \sin \left(\theta_{d}\right) & \cos \left(\theta_{d}\right)
\end{array}\right] .
\end{aligned}
$$

Equation (2) shows that there is a translation and a generalized rotation between two adjacent $\mathrm{RD}$ images. With this linear mapping relationship, a method based on the concept of rotation correlation was proposed to obtain the RV in [17]. However, the accuracy of RV estimation may be affected by the unknown RC. Later, the authors in [18] proposed to use two-dimensional (2D) Fourier transform (FT) and polar mapping to eliminate the impact of the target's unknown RC. The 2D FT is defined by

$$
F(u, v)=\int_{-\infty}^{\infty} \int_{-\infty}^{\infty} I(x, y) e^{-j 2 \pi u x} e^{-j 2 \pi v y} d x d y
$$

where $F(u, v)$ is the $2 \mathrm{D}$ FT of the image $I(x, y)$. 
Let $I_{k}(x, y)$ be the RD image formed around $t_{m k}, k=1,2$, and $F_{k}(u, v)$ is the relative $2 \mathrm{D}$ spectrum. Then, based on the linearity of FT, we will get

$$
\begin{aligned}
F_{2}(u, v)= & F_{1}\left((u, v) H\left(\theta_{d}\right)\right) \\
& \cdot \exp \left(-j 2 \pi(u, v) H\left(\theta_{d}\right)\left(X_{o}^{\prime}, Y^{\prime}{ }_{o}\right)^{T}\right) .
\end{aligned}
$$

By taking amplitudes of the spectrum on two sides, the influence of unknown RC is eliminated; that is,

$$
\left|F_{2}(u, v)\right|=\left|F_{1}\left((u, v) H\left(\theta_{d}\right)\right)\right| .
$$

The normalized power spectrum is defined as follows:

$$
\varepsilon_{k}(u, v)=\frac{\left|F_{k}(u, v)\right|^{2}}{\left|F_{k}(0,0)\right|^{2}}, \quad k=1,2 .
$$

Then, in the polar coordinate system, $\rho=\sqrt{u^{2}+v^{2}}, \theta=$ $\arctan (v / u)$, the normalized energies become

$$
\bar{\varepsilon}_{2}(\rho, \theta)=\bar{\varepsilon}_{1}(\lambda(\theta ; H) \rho, \varphi(\theta ; H)),
$$

where

$$
\begin{aligned}
& \bar{\varepsilon}_{k}(\rho, \theta)=\varepsilon_{k}(\rho \sin \theta, \rho \cos \theta), \quad k=1,2, \\
& \lambda(\theta ; H)=\left(\left(h_{11} \cos \theta+h_{21} \sin \theta\right)^{2}\right. \\
& \left.\quad+\left(h_{12} \cos \theta+h_{22} \sin \theta\right)^{2}\right)^{1 / 2}, \\
& \varphi(\theta ; H)=\arctan \left(\frac{h_{12} \cos \theta+h_{22} \sin \theta}{h_{11} \cos \theta+h_{21} \sin \theta}\right) .
\end{aligned}
$$

Here, $\lambda(\theta ; H)$ may be treated as a SF in the radial direction and $\varphi(\theta ; H)$ represents the relative angular displacement. If $H\left(\theta_{d}\right)$ is a standard rotation matrix, which means $\eta_{r} / \eta_{a}=1$, it is easy to show that $\lambda(\theta ; H)=1$, for all $\theta$, and $\varphi(\theta ; H)=$ $\theta+\theta_{d}$ is a simple translation. Therefore, the target's rotation in image domain was converted to only translation on the polar spectrum images. The same idea was used in [18] for the estimation of RV, although the relationship between two polar images is not given directly like (8). However, the computation is still not so efficient for the $2 \mathrm{D}$ interpolation which is required in each iteration. Further, when the aspect angle is relatively large, the two subaperture images may be defocused and the performance of the RV estimation may be decreased. Still, no information about the RC is given by these two methods, and thus this may hamper focused image forming with large aspect angle. Based on this, three main improvements are proposed in this paper:

(1) The keystone transform is introduced in the preprocessing step to get better focused subaperture images.

(2) The radial projection is introduced for the estimation of $\mathrm{RV}$, and the time cost 2D image interpolation and correlation operations are avoided.

(3) With the estimated RV, a method is proposed to search the RC based on minimum entropy criterion, and both the RV and RC may be provided for focused image forming with large aspect angle.

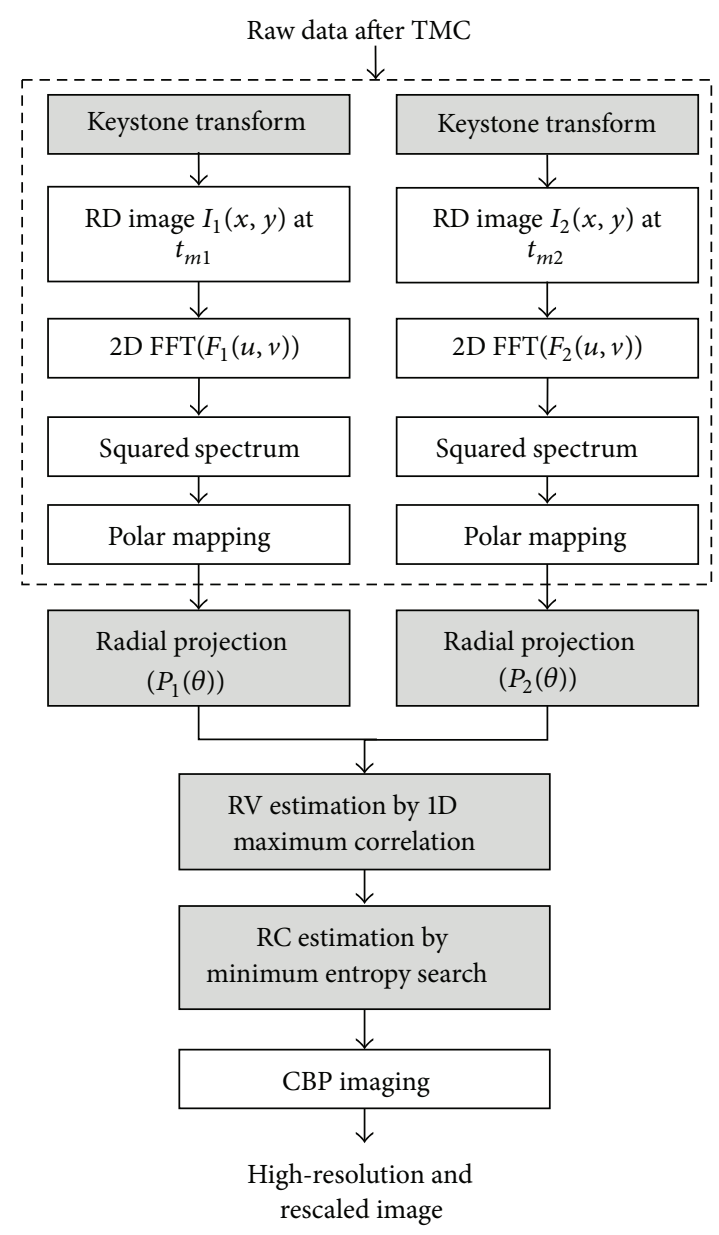

FIGURE 2: Proposed method for rotation estimation.

A block diagram of the whole procedure for rotation estimation is illustrated in Figure 2, and the main differences compared with $[17,18]$ are shown with another color.

\section{The Improved Rotation Parameters Estimation Method}

3.1. RV Estimation Based on Radial Projection. Before the $\mathrm{RD}$ image formation of two subapertures, the keystone processing [19] is used to compensate the linear range migration through resolution cells, in order to get more focused subimages. Then, the two neighboring subimages are Fourier transformed, normalized, and polar mapped according to (4), (7), and (8).

To make the computation more efficient, we introduce the radial projection for the estimation of RV. Radial projection is proved to be an efficient and robust algorithm for the estimation of 2D affine transformations in frequency domain [20]. Here, the $H\left(\theta_{d}\right)$ can be treated as the affine transformation matrix between two neighboring RD images, and it is even more simple compared with the case of [20], because it is 
determined by only one variable, while the transformation matrix in [20] has four degrees of freedom:

$$
\begin{aligned}
P\left\{Q_{d n} \leq T_{d}\right\} & =P\left\{\Lambda_{01} \leq T_{d}, \Lambda_{02} \leq T_{d}\right\} \\
& =\left(P\left\{\Lambda \leq T_{d}\right\}\right)^{2}=\left(P_{f a, 1}\right)^{2}, \\
P\left\{Q_{d} \leq T_{d}\right\} & =1-P\left\{Q_{d} \geq T_{d}\right\} \\
& =1-\left(P\left\{Q_{d n} \geq T_{d}\right\}\right)^{N} \\
& =1-\left[1-\left(P_{f a, 1}\right)^{2}\right]^{N}=P_{f a, d} .
\end{aligned}
$$

The radial projection is defined as

$$
P_{k}(\theta)=\int_{0}^{\infty} \rho \bar{\varepsilon}_{k}(\rho, \theta) d \rho, \quad k=1,2
$$

and the relationship between two projections is determined by

$$
P_{2}(\theta)=\frac{1}{\lambda^{2}(\theta ; H)} P_{1}(\varphi(\theta ; H)) .
$$

According to (12), the RV can be estimated by solving a maximum correlation problem; that is,

$$
\begin{aligned}
& \widehat{\omega}_{o}=\underset{\omega_{o}}{\arg \max } C\left(P_{2}(\theta), \frac{1}{\lambda^{2}\left(\theta ; H\left(\theta_{d}\right)\right)}\right. \\
& \left.\cdot P_{1}\left(\varphi\left(\theta ; H\left(\theta_{d}\right)\right)\right)\right)
\end{aligned}
$$

where $C(\cdot)$ means the function of correlation coefficient, and we will replace it with $f\left(\omega_{o}\right)$ in the following sections for convenience.

In (13), $P_{1}\left(\varphi\left(\theta ; H\left(\theta_{d}\right)\right)\right)$ may be calculated numerically using interpolation method. Here, we propose to approximate $P_{1}(\theta)$ using cosine series to make the computation more efficient without loss of accuracy. $P_{1}(\theta)$ is a periodic function with period, so it can be approximated as

$$
P_{1}(\theta) \simeq \widetilde{P}_{1}(\theta)=\gamma_{0}+\sum_{m=1}^{M_{0}} \gamma_{m} \cos \left(2 m \theta-\phi_{m}\right),
$$

where

$$
\begin{aligned}
& \alpha_{m}=\frac{2}{\pi} \int_{0}^{\pi} P_{1}(\theta) \cos (2 m \theta) d \theta, \quad m=0,1, \ldots, M_{0} \\
& \beta_{m}=\frac{2}{\pi} \int_{0}^{\pi} P_{1}(\theta) \sin (2 m \theta) d \theta, \quad m=1,2, \ldots, M_{0} \\
& \phi_{m}=\arctan \frac{\beta_{m}}{\alpha_{m}} \\
& \gamma_{m}=\frac{\alpha_{m}}{\cos \left(\phi_{m}\right)}, \\
& \gamma_{0}=\frac{\alpha_{m}}{2}
\end{aligned}
$$

$M_{0}$ is the number of terms used in the cosine series. Usually, 30-40 cosine series could have been used without any loss of accuracy. Here, we set $M_{0}=40$.

By combining (13) and (14), it is possible to solve the gradient of $f\left(\omega_{0}\right)$, but the optimization method based on gradient is not recommended. Here, the Nelder-Mead approach [21] is used to solve the optimization of (13), for it is a very effective line search method. Although an arbitrary initialization may be enough for the Nelder-Mead approach, a better start point can greatly reduce the number of iterations. Assuming the $H\left(\theta_{d}\right)$ to be a standard rotational matrix, there will be a simple translation between $P_{1}(\theta)$ and $P_{2}(\theta)$. If the radial projections in (11) are computed at increments $\Delta \theta=$ $\pi / N_{0}$ (in our experiments, $N_{0}=360$ ) over $[0, \pi)$, then, $\omega_{o}$ can be initialized using

$$
\begin{aligned}
& k=\arg \max _{k}\left[\operatorname{IFT}_{\theta}\left(\left(\mathrm{FT}_{\theta}\left(P_{1}\right)\right)^{*} \mathrm{FT}_{\theta}\left(P_{2}\right)\right)(k)\right], \\
& \omega_{o \text { _Init }}=\frac{k \Delta \theta}{\left(t_{m 2}-t_{m 1}\right)} \text {, }
\end{aligned}
$$

where $\mathrm{FT}_{\theta}()$ and $\operatorname{IFT}_{\theta}()$ are the $1 \mathrm{D}$ FT and inverse FT in the $\theta$-direction.

In the total procedure for the estimation of $\mathrm{RV}$, the $2 \mathrm{D}$ interpolation is done only once in the polar coordinate system, and the $2 \mathrm{D}$ maximum correlation problem is converted to a 1D polar curve matching problem according to (13), which can be solved in a few numbers of iterations efficiently, so the computation burden is greatly reduced here.

3.2. RC Estimation Based on Entropy Minimization. When echoes are collected from a relatively large aspect angle interval in wideband radar imaging system, the target's MTRC cannot be ignored. In order to apply the delicate imaging algorithm like CBP for high-resolution imaging, not only the RV but also the RC should be known. The commonly used autofocusing methods are not able to locate the target's equivalent $\mathrm{RC}$ in the RD images. Generally speaking, the range bias of $\mathrm{RC}$ is caused by the range alignment and the unknown reference point. It is usually difficult to estimate the $\mathrm{RC}$ directly. However, on the basis of effective RV estimation, the range bias of $\mathrm{RC}$ may be obtained by $1 \mathrm{D}$ searching directly.

After range compression and TMC, the radar echoes related to a single scatter $P\left(x_{o}, y_{o}\right)$ take the following form:

$$
\begin{aligned}
s\left(r_{n}, t_{m}\right)= & A_{o} \operatorname{sinc}\left(\frac{2 B}{c}\left(r_{n}-r_{o}\left(t_{m}\right)\right)\right) \\
& \cdot \exp \left(-j \frac{4 \pi f_{c}}{c} r_{o}\left(t_{m}\right)\right),
\end{aligned}
$$

where $A_{o}$ is the scattering coefficient, $B$ is the bandwidth of the transmitted pulse, $f_{c}$ is the carrier frequency, $r_{n}$ is the sampling position in range direction, and $r_{o}\left(t_{m}\right)$ is the relative distance between the radar and the scattering point; that is,

$$
r_{o}\left(t_{m}\right)=r_{c}-x_{o} \cos \left(\omega_{o} t_{m}\right)+y_{o} \sin \left(\omega_{o} t_{m}\right),
$$

where $r_{c}$ is the residual range bias of RC after TMC. 
Taking the second-order approximation of (18) and substituting it into (17),

$$
\begin{aligned}
s\left(r_{n}, t_{m}\right)= & A_{o} \operatorname{sinc}\left(\frac{2 B}{c}\left(r_{n}-r_{c}+x_{o}-r_{o}^{\prime}\left(t_{m}\right)\right)\right) \\
& \cdot \exp \left(j \varphi_{o}\right) \exp \left(-j \frac{4 \pi f_{c}}{c} r_{o}^{\prime}\left(t_{m}\right)\right),
\end{aligned}
$$

where $r_{o}^{\prime}\left(t_{m}\right)=y_{o} \omega_{o} t_{m}+(1 / 2) x_{o} \omega_{o}^{2} t_{m}^{2}$ is the scatter's migration during CPI and $\varphi_{o}$ is a constant phase. The secondorder phase component of (19) will cause a Doppler modulation and resulting blurring in cross-range direction. To compensate this rotating phase, the received echoes in (19) are shifted in range direction by $\tau$ and multiplied with the corresponding secondary phase compensation factor; that is,

$$
s_{\tau}\left(r_{n}, t_{m}\right)=s\left(r_{n}+\tau, t_{m}\right) \exp \left(j \frac{4 \pi f_{c}}{c} \frac{1}{2} r_{n} \omega_{o}^{2} t_{m}^{2}\right) .
$$

The range position of $\mathrm{RC}$ is then estimated by the following:

$$
\widehat{r}_{c}=\min _{\tau} \operatorname{IE}\left\{\left|\mathrm{FT}_{t_{m}}\left(s_{\tau}\left(r_{n}, t_{m}\right)\right)\right|\right\},
$$

where $\mathrm{FT}_{t_{m}}$ ( ) means FT in the cross-range direction to form the $\mathrm{RD}$ image, $\mathrm{IE}($ ) represents the $2 \mathrm{D}$ entropy function of the radar image [22], and the minimization of (21) can be solved by a simple line search in range direction.

With all the rotation parameters known, the CBP algorithm is then used to generate a high-resolution and rescaled image. The traditional CBP can be accelerated using GPU parallelization [23].

\section{Simulations and Analysis}

This section demonstrates the effectiveness and robustness of the proposed algorithm using both simulated and some collected ISAR data, and the algorithm proposed by [18] is taken for comparison during the RV estimation.

\subsection{Simulation Results Using Point Scattering Model}

4.1.1. $R V$ and $R C$ Estimation Results. In the numerical simulation, a 3D target which contains 330 isolated scattering points is used. The $3 \mathrm{D}$ model of the target and its three projected views are shown in Figure 3. The target is located $10 \mathrm{~km}$ away from the origin on the $y$-axis and moves along a straight line with speeds $V_{x}=151.03 \mathrm{~m} / \mathrm{s}, V_{y}=87.20 \mathrm{~m} / \mathrm{s}$, and $V_{z}=$ $0 \mathrm{~m} / \mathrm{s}$. The simulated ISAR system transmits 400 chirp signals with $800-\mathrm{MHz}$ bandwidth per second. The carrier frequency is $9.15 \mathrm{GHz}$, and the echoes are coherently demodulated and inphase/quadrature phase channel- (I/Q-) sampled with a rate of $1 \mathrm{GHz}$ for pulse compression. The data collection time is $10.24 \mathrm{~s}$, and during this time interval, the rotated angle is $8.8^{\circ}$, and the resulting $\mathrm{RV}$ is $0.015 \mathrm{rad} / \mathrm{s}$. The simulated raw data is shown in Figure 4(a), the TMC is taken by minimum entropy methods [22], and the range compressed data after TMC is shown in Figure 4(b).
By dividing the received data equally into two parts and taking the keystone transform, the $\mathrm{RD}$ images are formed as in Figures 5(a) and 5(b). The corresponding squared FT magnitudes are shown in Figures 5(c) and 5(d). There is a clear rotation among the two radial projections (Figure 5(e)). $P_{1}(\theta)$ is approximated using 40 terms of cosine series (Figure 5(f)).

The RV is initialized to be $0.0124 \mathrm{rad} / \mathrm{s}$ using (16), and it takes 11 iterations to reach the maximum point (Figure 6(a)), respectively; the RV is estimated to be $0.0149 \mathrm{rad} / \mathrm{s}$, which is very close to the theoretical value. Before estimating the $\mathrm{RC}$, the image center of gravity is first moved to the zero point in $\mathrm{RD}$ domain to reduce the searching area; then, the range position of RC is searched by entropy minimization from $-15 \eta_{r}$ to $15 \eta_{r}$ with a step of $\eta_{r}$. Finally, the RC is estimated to be $\widehat{r}_{c}=3.15 \mathrm{~m}$ (Figure 6(b)). With all the rotational parameters, the CBP algorithm is taken and the result is shown in Figure 6(d). Compared with the RD imaging result in Figure 6(c), the energy of scattering point in Figure 6(d) is well focused, and the size of the target is properly rescaled.

4.1.2. Performance Analysis. Since the estimation of RC is directly related to the result of RV estimation, this section only illustrates the effectiveness of the proposed RV estimation. The root mean square error (RMSE) of the proposed RV estimation method against the signal-to-noise ratio (SNR) is presented in Figure 7, and the RMSE curve of the proposed method in [18] is presented for comparison. It is shown that the proposed method outperforms the method in [18] for low SNR scenarios when radial projection is introduced for RV estimation, partially because the radial projection may be viewed as a feature extraction process with a weighting operator and thus may suppress the impact of the noise. What is more, the performance of RV estimation gets better when keystone processing is included in the rotation estimation scheme, as in Figure 7, because keystone processing compensates for the range migration of scattering centers in each RD image. Thus, an integrated RV estimation scheme in the proposed method includes both the keystone processing and radial projection.

Then, the performance of proposed RV estimation method is compared with the method in [18] when the image forming plane is not so stationary, which may occur for wideangle ISAR imaging applications. In the first case, the target is rotating with a constant acceleration, where the central $\mathrm{RV}$ is set to be $0.015 \mathrm{rad} / \mathrm{s}$. The RMSE of the RV estimation against different rotational acceleration, which varies from $10^{-5} \mathrm{rad} / \mathrm{s}^{2}$ to $10^{-4} \mathrm{rad} / \mathrm{s}^{2}$, is shown in Figure 8(a). It can be seen from Figure 8(a) that the RV estimation gets worse for both methods when the rotational acceleration gets larger because the focusing quality of each $\mathrm{RD}$ image degrades. However, the proposed method still outperforms the method in [18]. In the above analysis, the imaging plane is considered to be planar. In the second case, the imaging plane is disturbed with a periodic error. The period of the disturbance is set to be $2 \mathrm{~s}$, while the amplitude of the disturbance varies from $0.2 \mathrm{deg}$. to $2 \mathrm{deg}$. The RMSE of the RV estimation 

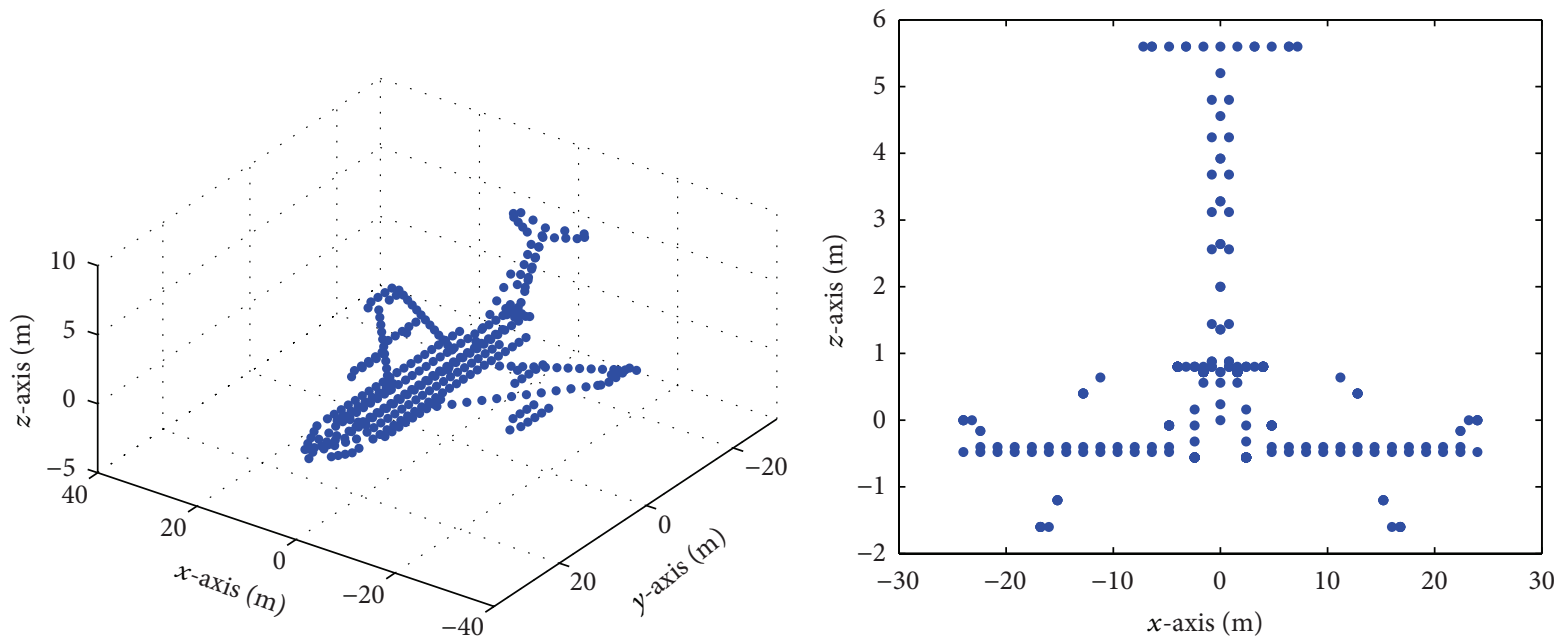

(a)

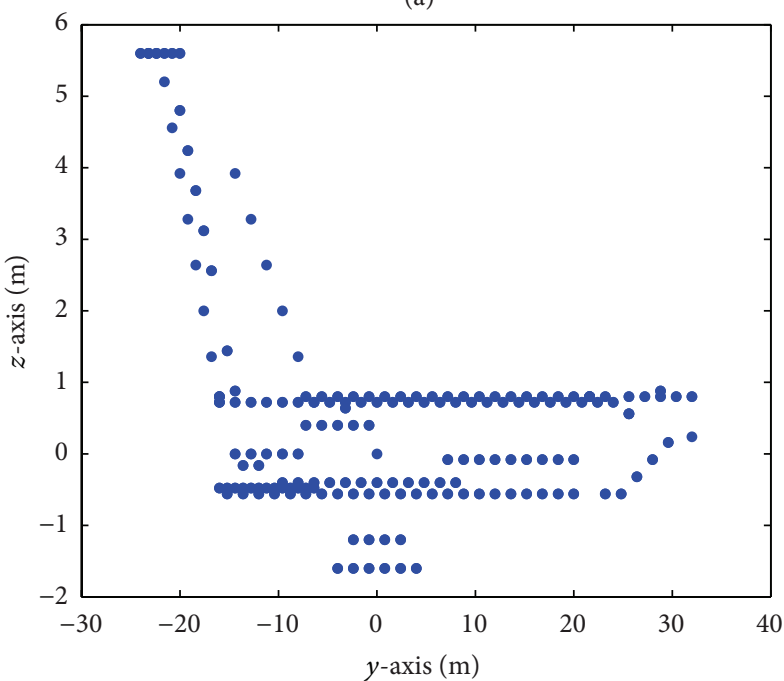

(b)

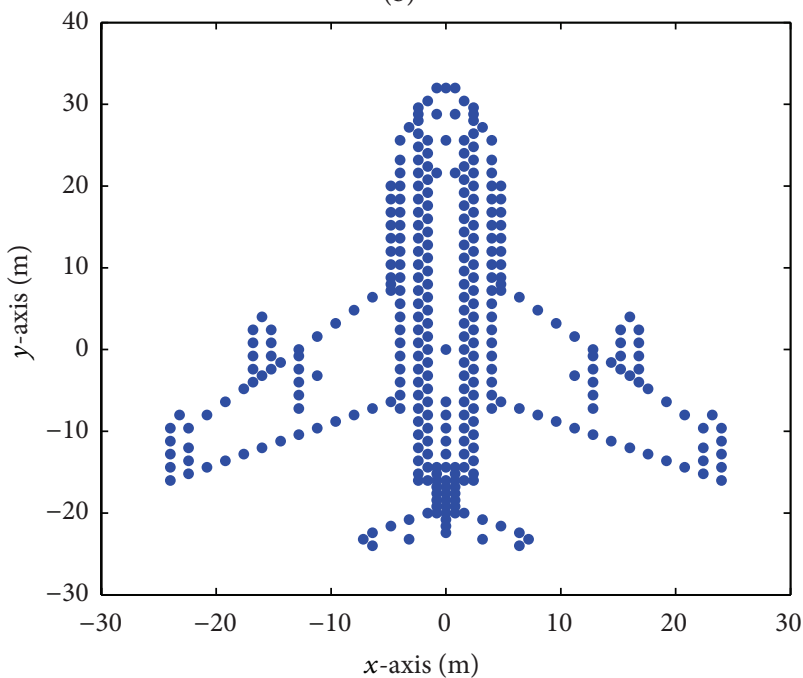

(d)

FIGURE 3: Three-dimensional model of the target, consisting of 330 points. (a) The three-dimensional distribution of the scattering points. (b) Front view. (c) Side view. (d) Top view.

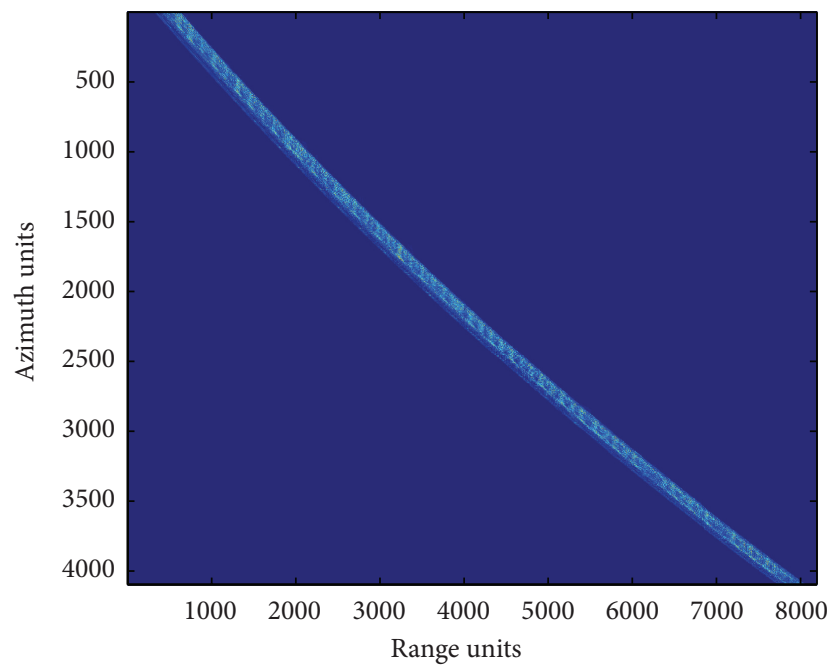

(a)

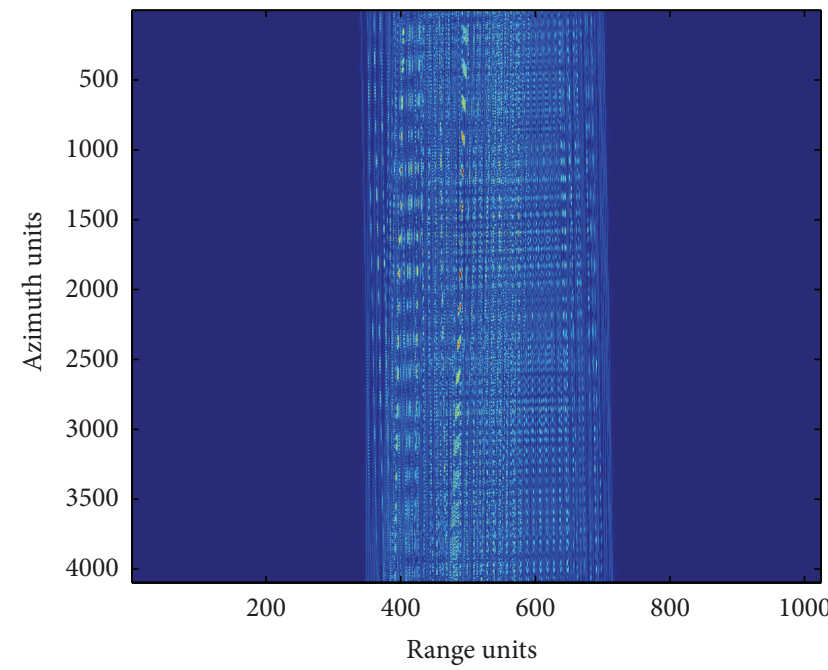

(b)

FIgURE 4: The simulated raw data and the TMC results. (a) The simulated raw data with line motion. (b) The received data after motion compensation. 


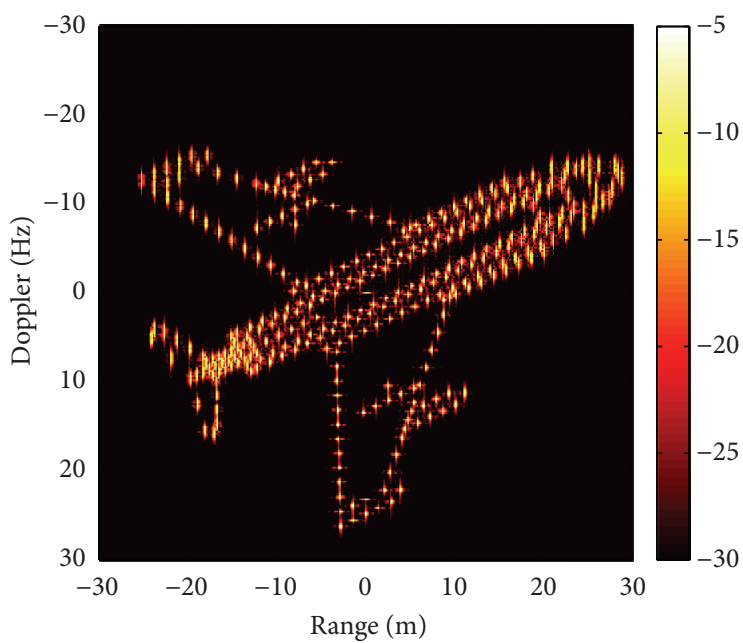

(a)

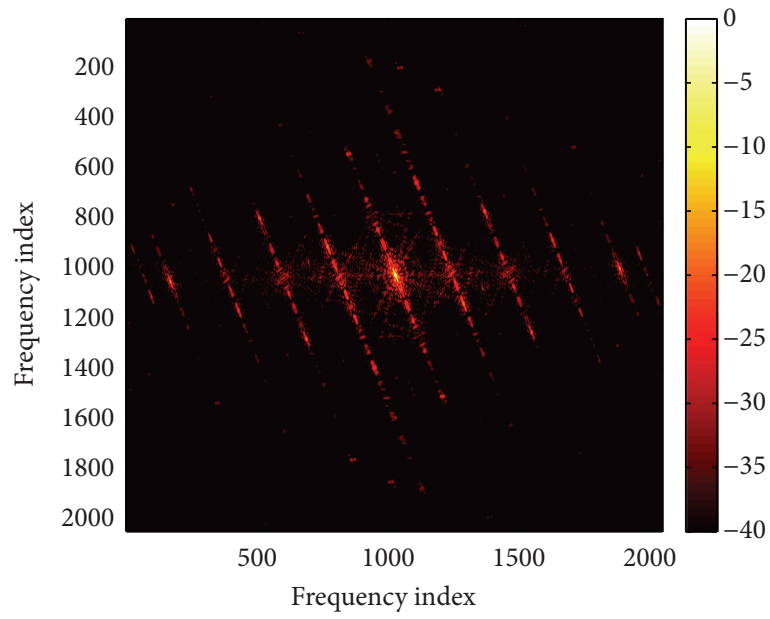

(c)

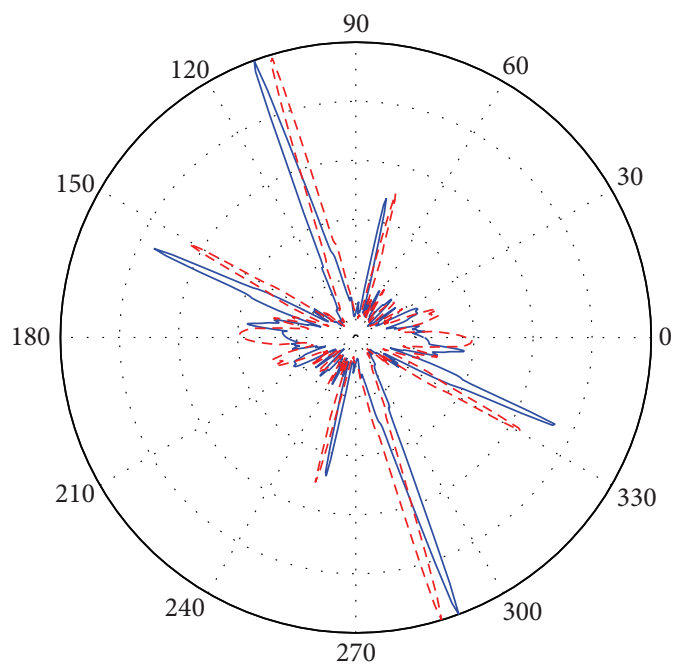

$-P_{1}(\theta)$

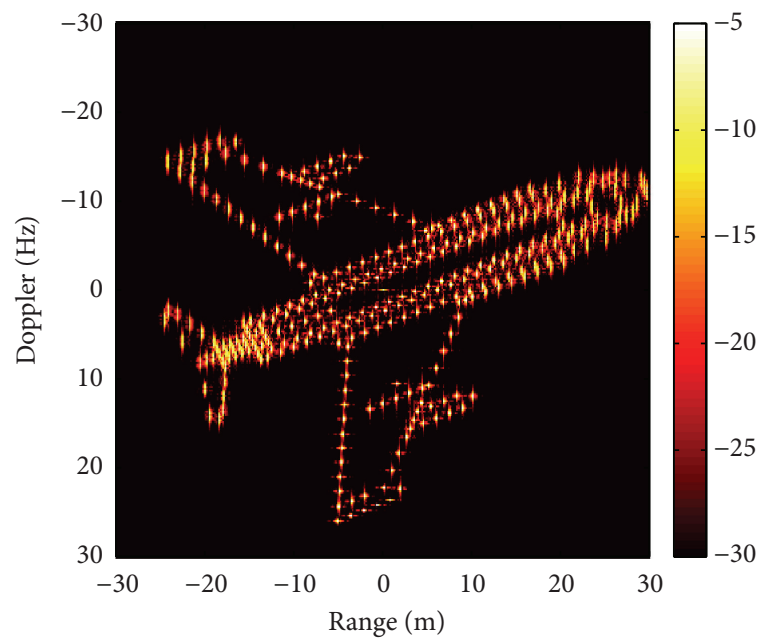

(b)

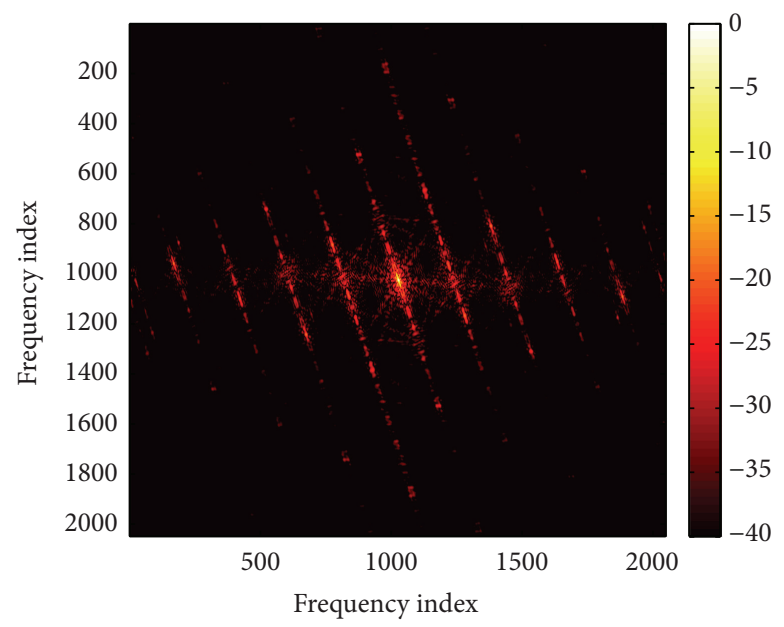

(d)

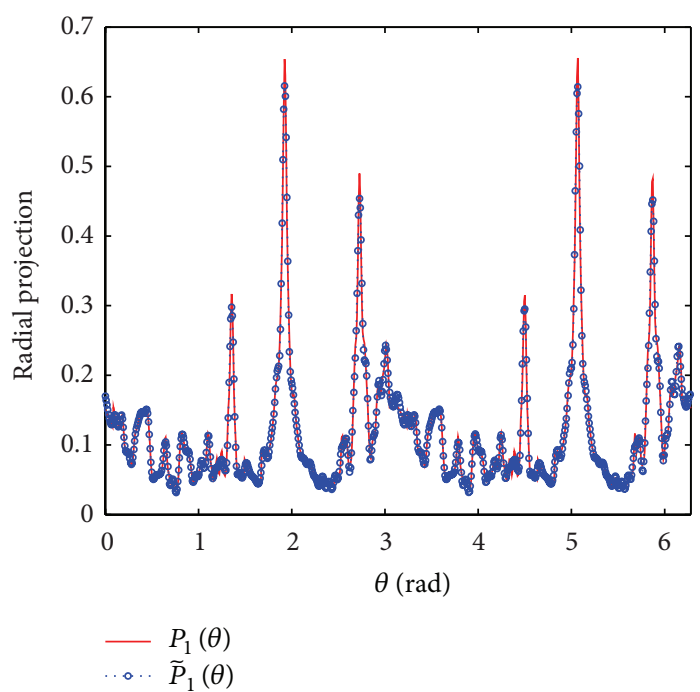

(f)

FIgURE 5: Experiments with simulated data (I). (a) RD image of 1-2048 echoes (dB). (b) RD image of 2049-4096 echoes (dB). (c) Squared FT magnitude relative to (a) (dB). (d) Squared FT magnitude relative to (b) (dB). (e) Radial projections. (f) $P_{1}(\theta)$ and its approximation with 40 terms of cosine series. 


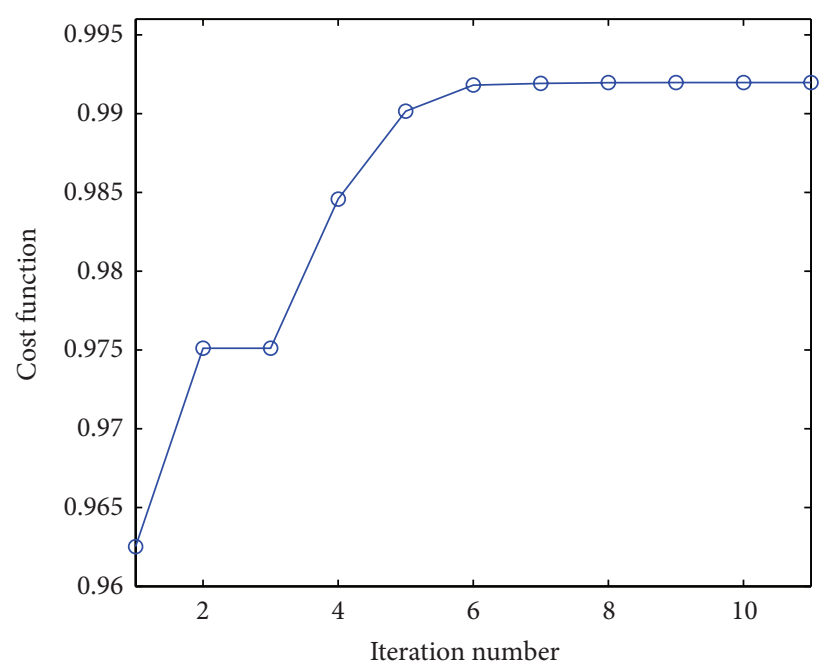

(a)

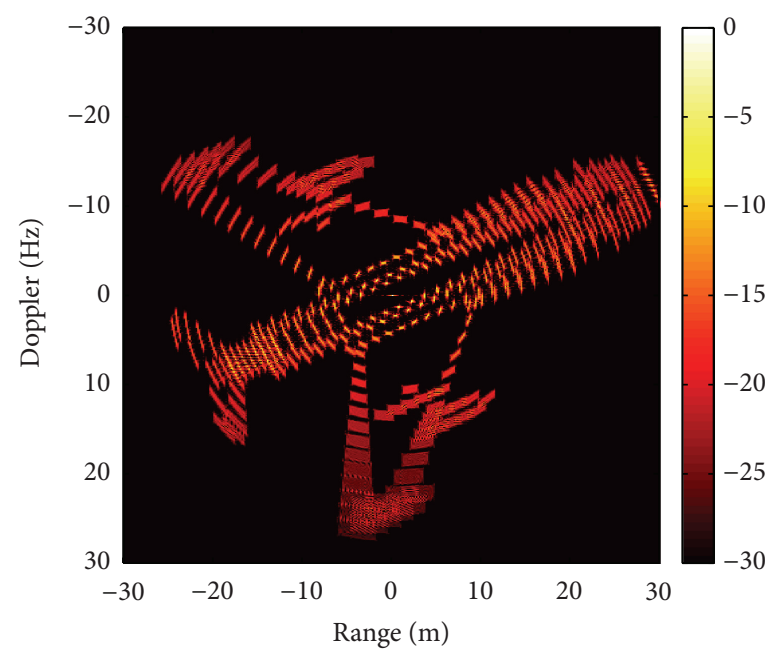

(c)

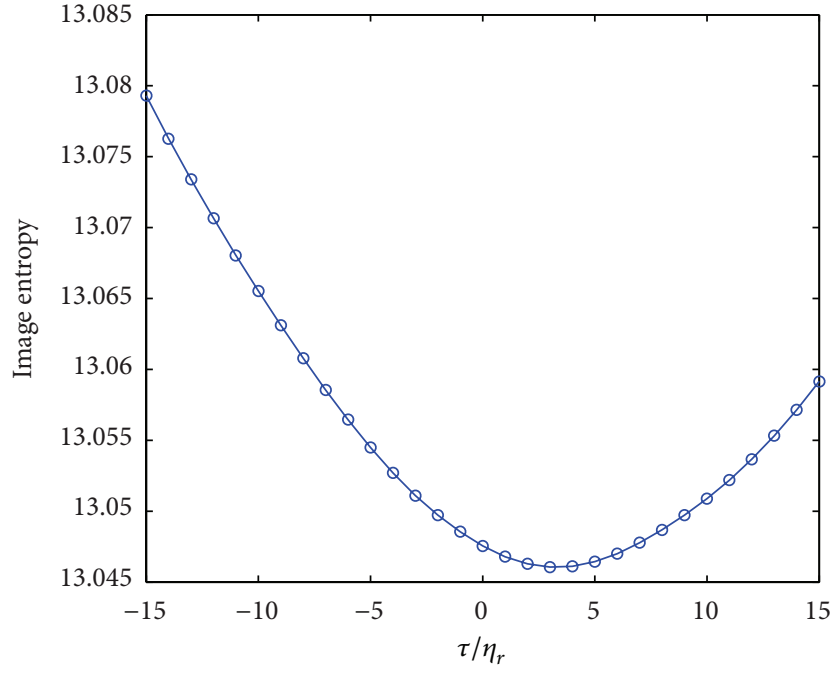

(b)

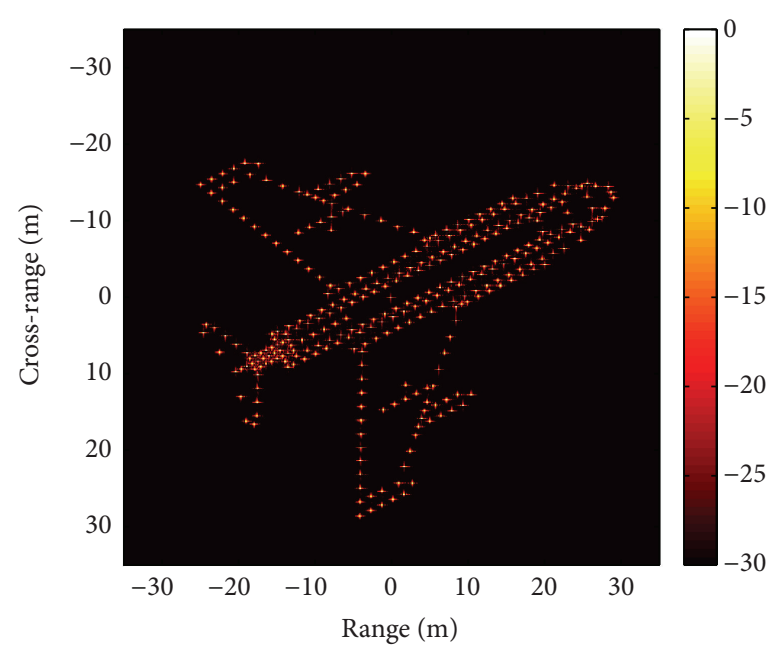

(d)

FIgURE 6: Experiments with simulated data (II). (a) The iteration steps of Nelder-Mead algorithm. (b) The entropy of RD images when searching for RC. (c) RD image of total 4096 echoes (dB). (d) CBP imaging result (dB).

is presented in Figure 8(b), and the performance degrades when the disturbance gets serious. Still, the proposed RV estimation method is more robust.

\subsection{Simulation Results Using Collected Data. Experiments} with some real data of a Yak-42 airplane (see Figure 9) recorded by a C-band $(5.52 \mathrm{GHz})$ ISAR system $[17,22]$ are also used to demonstrate the effectiveness of the proposed method. This system transmits $400 \mathrm{MHz}$ chirp signals with $25.6 \mu$ s pulse width, and the target's echoes are dechirped and I/Q-sampled with a frequency of $10 \mathrm{MHz}$. The pulse repetition frequency is $400 \mathrm{~Hz}$, and a total of 4096 pulses are collected. These echoes are also equally divided to form two
RD images, as in Figures 10(a) and 10(b). The squared FT magnitudes are shown in Figures 10(c) and 10(d), and the corresponding radial projections are shown in Figure 11(a). $P_{1}(\theta)$ is approximated using 40 terms of cosine series (Figure 11(b)). The RV is initialized as $0.0043 \mathrm{rad} / \mathrm{s}$ and is finally estimated to be $0.0096 \mathrm{rad} / \mathrm{s}$ in 12 iterations (Figure 11(c)), corresponding to an aspect angle interval of $5.87 \mathrm{deg}$. The RC is estimated to be $3.373 \mathrm{~m}$ using the minimum entropy criterion, and the entropy curve is shown in Figure 11(d). The CBP image of Yak-42 (Figure 11(f)) is rescaled and more focused compared with the RD imaging result (Figure 11(e)), the length of line $A B$ is $36.094 \mathrm{~m}$, and $C D$ is $34.325 \mathrm{~m}$; both are very close to the real target contour. 


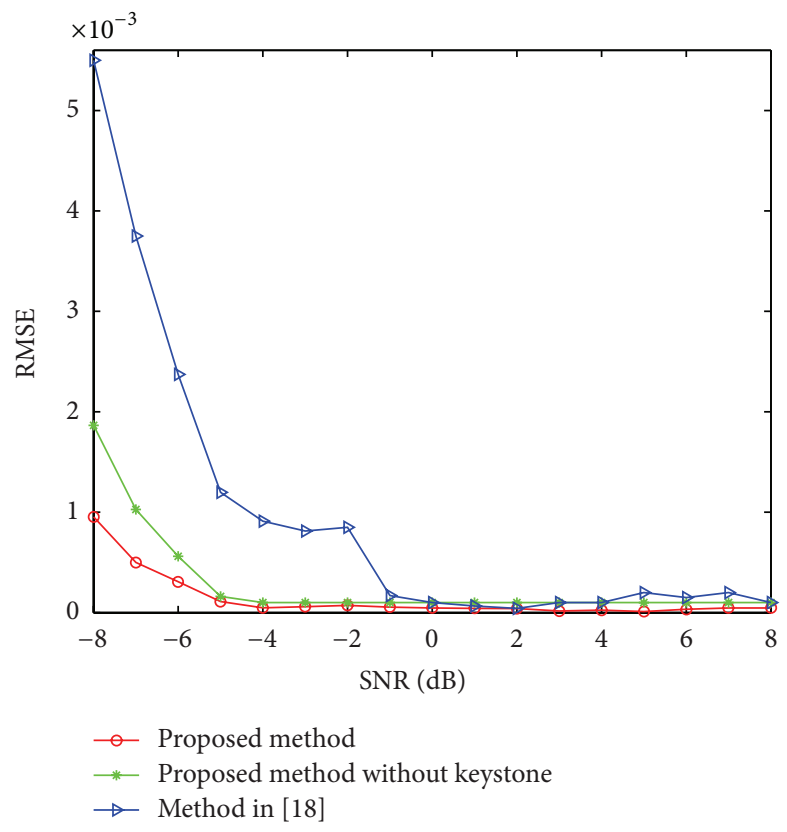

FIGURE 7: The RMSE of RV estimation versus SNR.

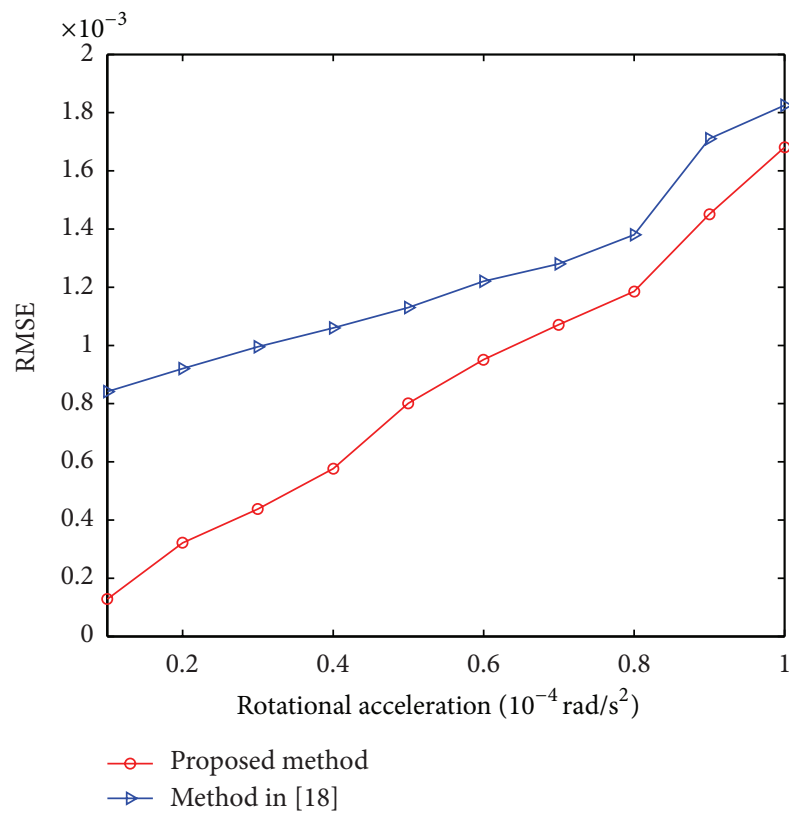

(a)

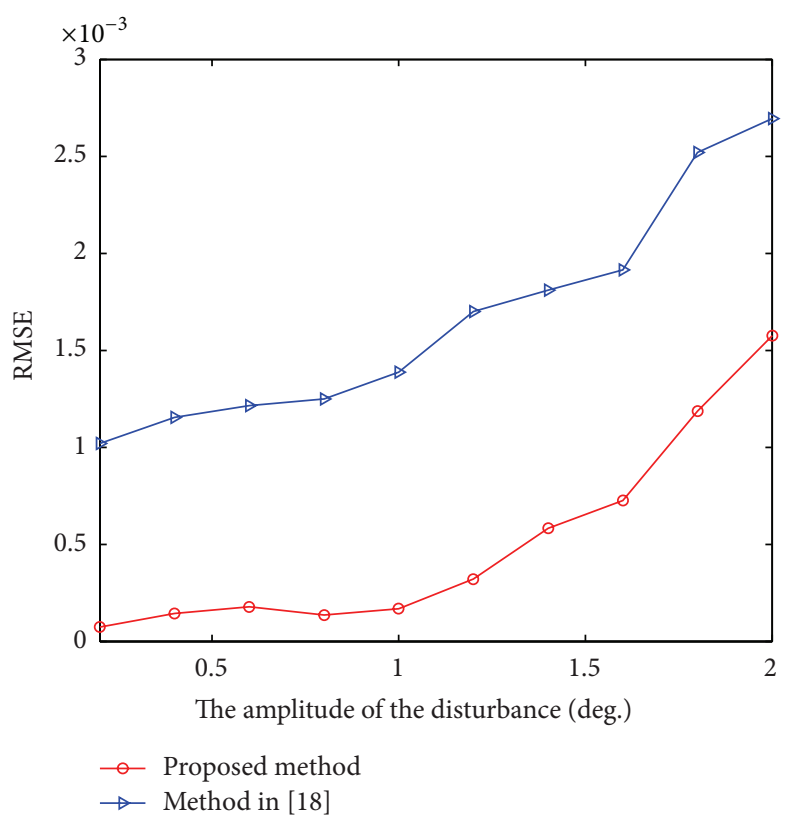

(b)

FIGURE 8: Robustness analysis in presence of nonuniform rotating. (a) The RMSE of RV estimation with different rotational acceleration. (b) The RMSE of RV estimation in presence of 3D rotation.

\section{Conclusion}

An improved method for estimating the target rotation parameters has been proposed in this paper. For the RV estimation, the keystone transform was used to compensate the linear range migration through resolution cells in subaperture. Then, the two adjacent $\mathrm{RD}$ images were processed by $2 \mathrm{D}$
FT to eliminate the influence of unknown RC, and the radial projection was applied to avoid the time-consuming process of $2 \mathrm{D}$ interpolation and image correlation. In addition, the $\mathrm{RV}$ was found by solving a $1 \mathrm{D}$ polar curve matching problem in a few iterations efficiently. Then, the influence of unknown $\mathrm{RC}$ on wideband ISAR imaging was studied in this paper. Based on the RV estimation, the target's RC was estimated 


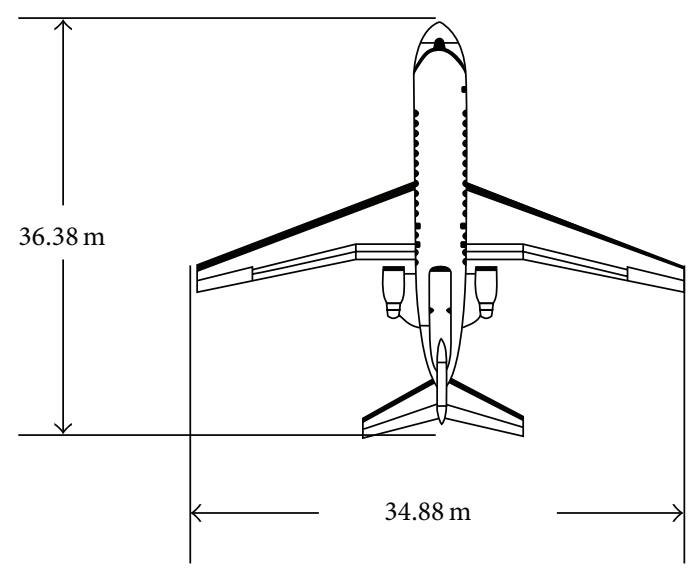

FIgURE 9: Graph of Yak-42.

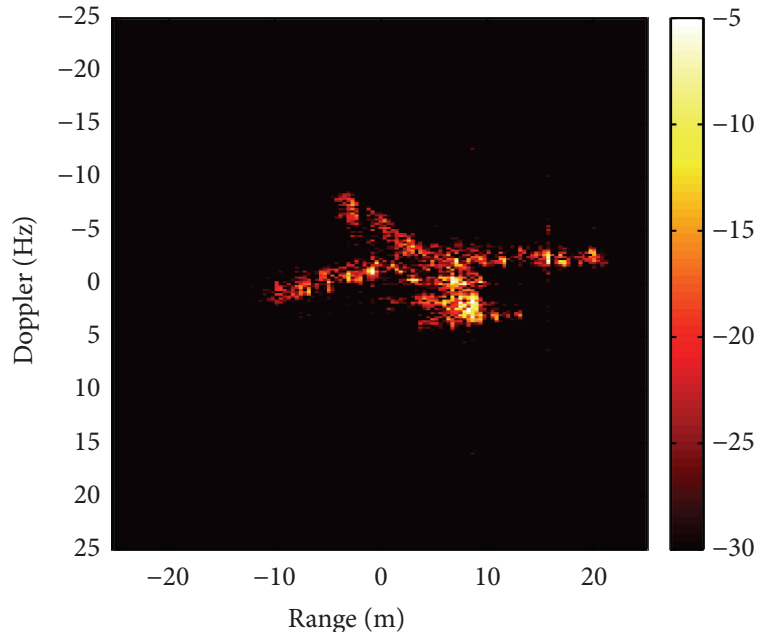

(a)

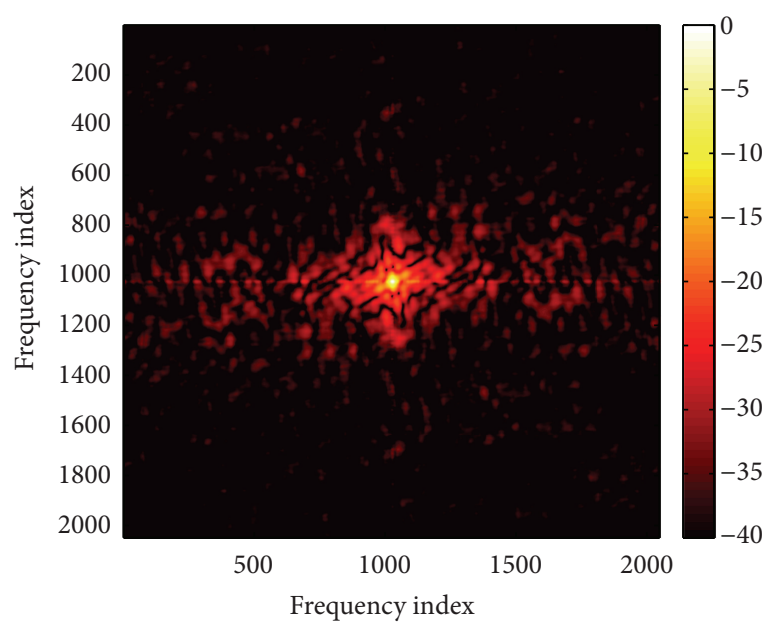

(c)

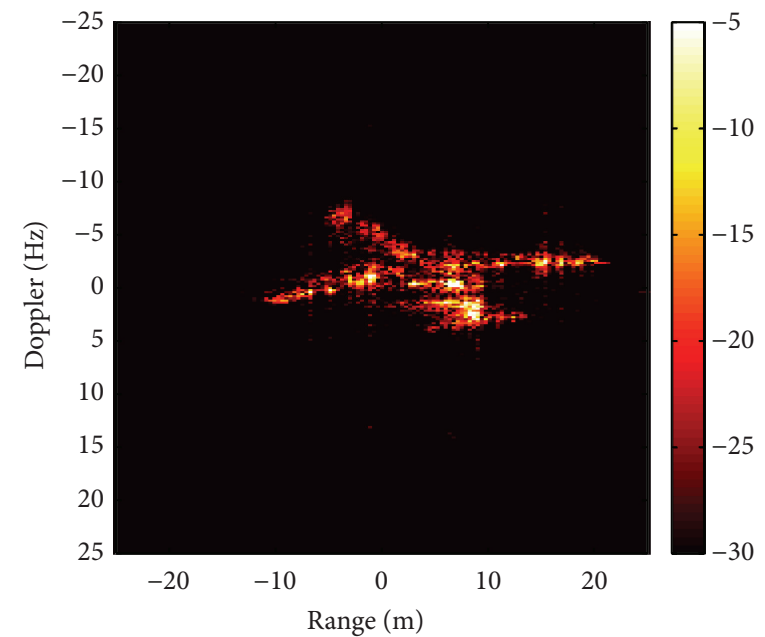

(b)

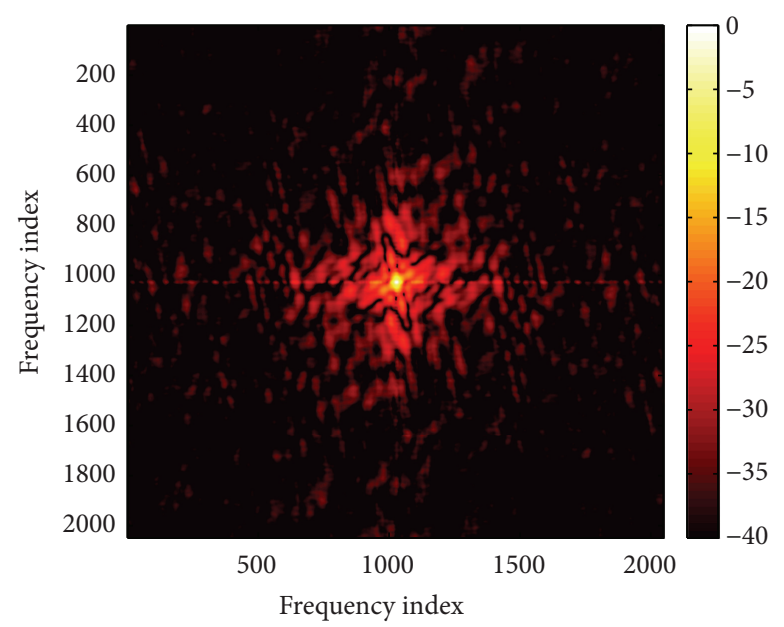

(d)

FIGURE 10: Experiments with real ISAR data (I). (a) RD image of 1-2048 echoes (dB). (b) RD image of 2049-4096 echoes (dB). (c) Squared FT magnitude relative to (a) (dB). (d) Squared FT magnitude relative to (b) (dB). 

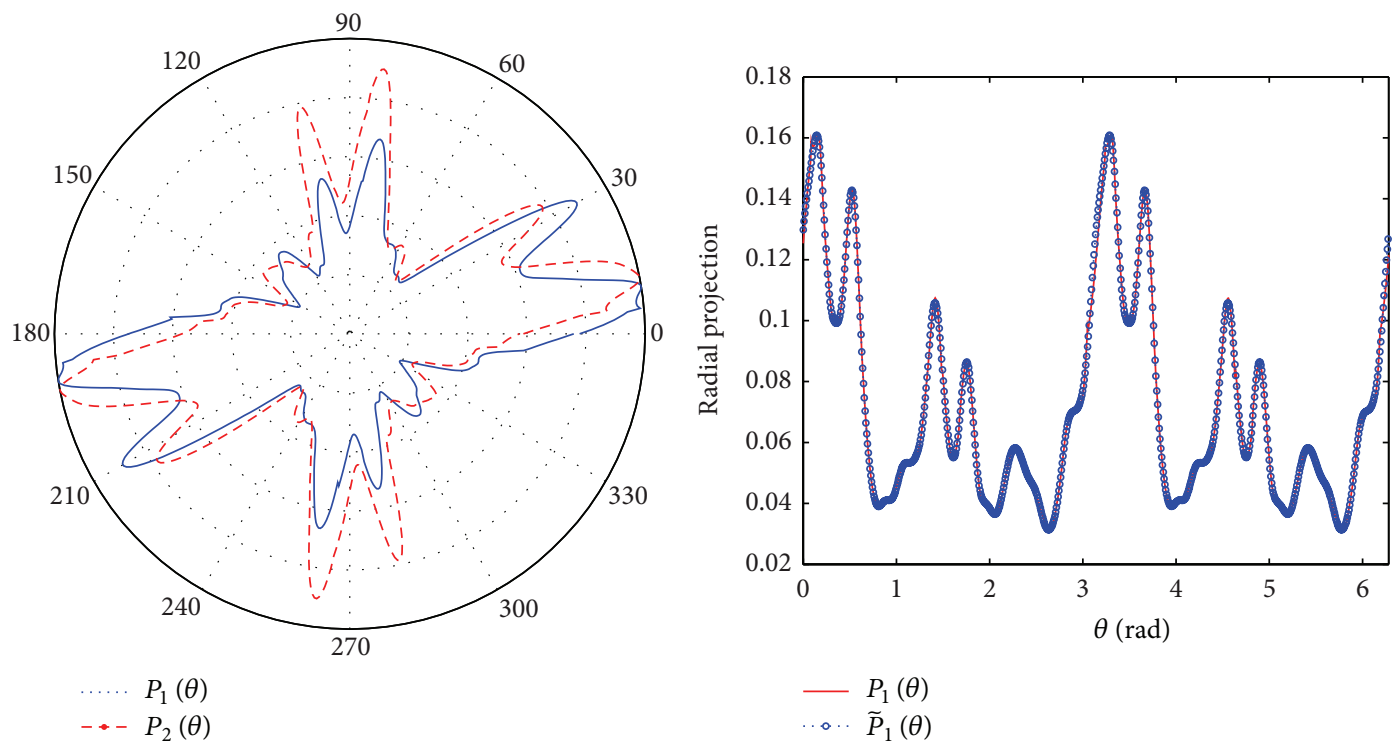

(a)

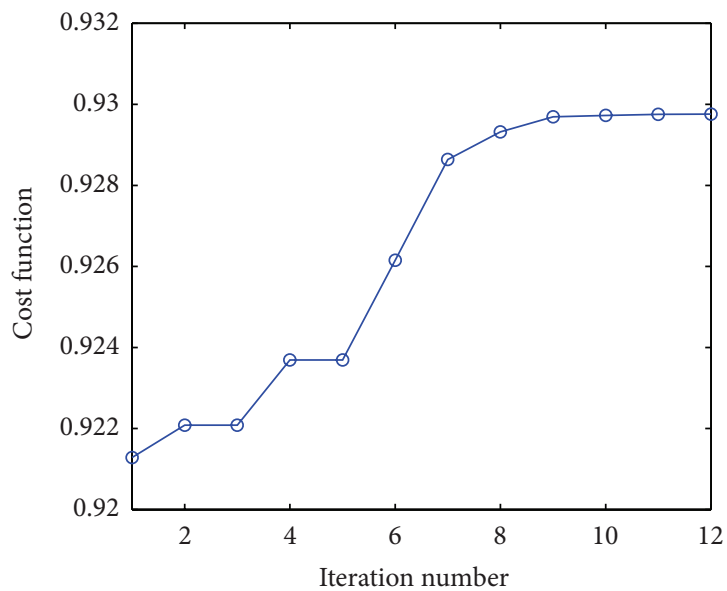

(c)

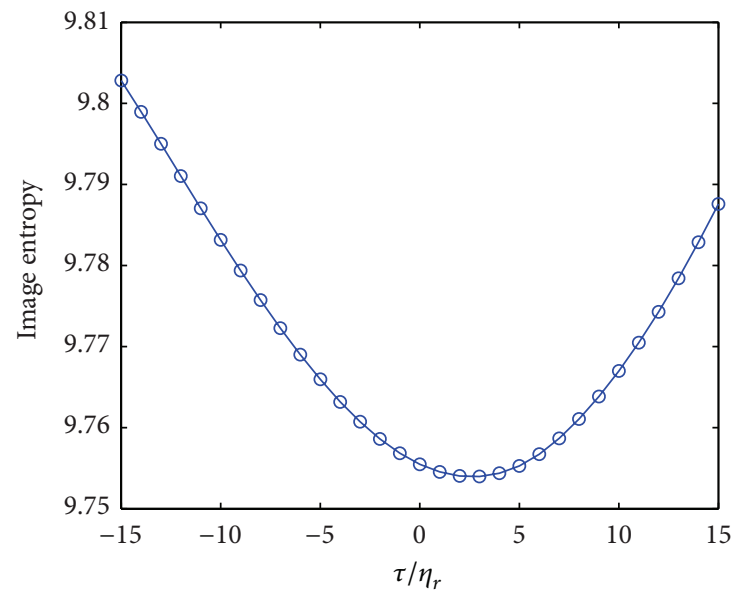

(d)

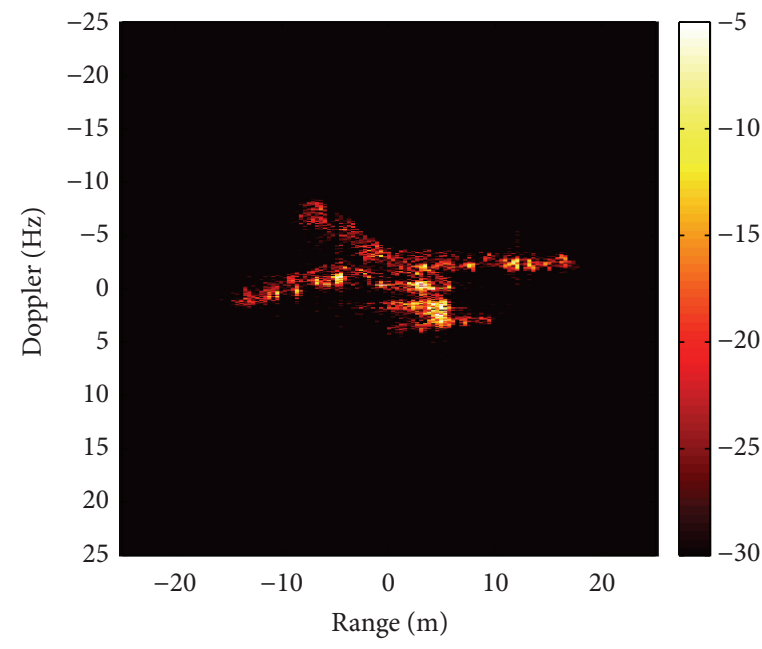

(e)

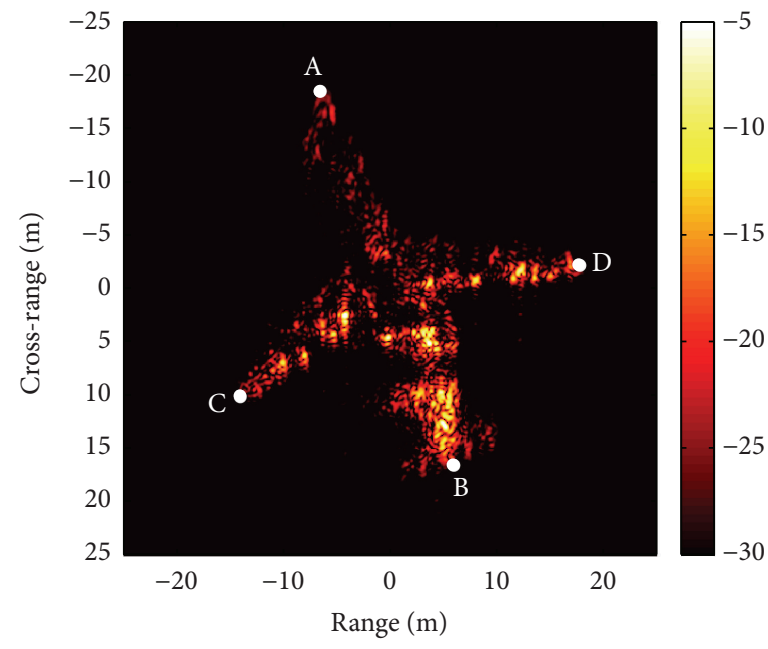

(f)

FIGURE 11: Experiments with real ISAR data (II). (a) Radial projections. (b) $P_{1}(\theta)$ and its approximation with 40 terms of cosine series. (c) The iteration steps of Nelder-Mead algorithm. (d) The entropy of RD images when searching for range bias of RC. (e) RD image of total 4096 echoes (dB). (f) CBP imaging of Yak-42 (dB). 
by a line search using minimum entropy criterion. With all the rotation parameters, the parallelized CBP algorithm was used to obtain the high-resolution and rescaled image.

In a simulation performed with known RV using 3D point scattering model, the target was properly rescaled and the scatters' MTRC were compensated very well. And the robustness of the proposed method has been validated with numerous experiments in presence of noise or nonuniform rotation. Furthermore, experiments with some collected data also demonstrated the effectiveness of the proposed algorithm, the CBP image of Yak-42 was well focused, and the contour of the target on image was very close to the real aircraft.

\section{Conflict of Interests}

The authors declare that there is no conflict of interests regarding the publication of this paper.

\section{Acknowledgments}

This work was in part supported by the NSFC (no. 61271417), in part supported by the major research plan of the NSFC (no. 61490693), and in part supported by the Research Foundation of Tsinghua University.

\section{References}

[1] C. C. Chen and H. C. Andrews, "Target-motion-induced radar imaging," IEEE Transactions on Aerospace and Electronic Systems, vol. 16, no. 1, pp. 2-14, 1980.

[2] M. Martorella, E. Giusti, L. Demi et al., "Target recognition by means of polarimetric ISAR images," IEEE Transactions on Aerospace and Electronic Systems, vol. 47, no. 1, pp. 225-239, 2011.

[3] S.-H. Park, M.-G. Joo, and K.-T. Kim, "Construction of ISAR training database for automatic target recognition," Journal of Electromagnetic Waves and Applications, vol. 25, no. 11-12, pp. 1493-1503, 2011.

[4] M. Martorella and F. Berizzi, "Time windowing for highly focused ISAR image reconstruction," IEEE Transactions on Aerospace and Electronic Systems, vol. 41, no. 3, pp. 992-1007, 2005.

[5] M. Y. Abdul Gaffar, W. A. J. Nel, and M. R. Inggs, "Selecting suitable coherent processing time window lengths for groundbased ISAR imaging of cooperative sea vessels," IEEE Transactions on Geoscience and Remote Sensing, vol. 47, no. 9, pp. 32313240, 2009.

[6] B. C. Flores and A. Ugarte, "Refinement of range-doppler imagery by feedback control," in Automatic Object Recognition III, vol. 1960, pp. 36-46, Orlando, Fla, USA, April 1993.

[7] Z. S. She and Z. D. Zhu, "An improved approach of cross-range scaling in ISAR," in Proceedings of the IEEE National Aerospace and Electronics Conference (NAECON '94), vol. 1, pp. 175-180, IEEE, Dayton, Ohio, USA, May 1994.

[8] Y. J. Huang, X. Wang, X. Li, and B. Moran, "Inverse synthetic aperture radar imaging using frame theory," IEEE Transactions on Signal Processing, vol. 60, no. 10, pp. 5191-5200, 2012.

[9] Y. Wang and Y. C. Jiang, "A novel algorithm for estimating the rotation angle in ISAR imaging," IEEE Geoscience and Remote Sensing Letters, vol. 5, no. 4, pp. 608-609, 2008.
[10] M. Martorella, "Novel approach for ISAR image cross-range scaling," IEEE Transactions on Aerospace and Electronic Systems, vol. 44, no. 1, pp. 281-294, 2008.

[11] Y. X. Wang, L. Hao, and V. C. Chen, "ISAR motion compensation via adaptive joint time-frequency technique," IEEE Transactions on Aerospace and Electronic Systems, vol. 34, no. 2, pp. 670-677, 1998.

[12] X. Li, H. Gu, and G. Liu, "A method for estimating the rotation angle of the ISAR image," Acta Electronica Sinica, vol. 28, no. 6, pp. 44-47, 2000.

[13] F. Prodi, "ISAR cross-range scaling using a correlation based functional," in Proceedings of the IEEE Radar Conference (RADAR '08), pp. 1-6, Rome, Italy, May 2008.

[14] J. M. Muñoz-Ferreras and F. Pérez-Martínez, "Non-uniform rotation rate estimation for ISAR in case of slant range migration induced by angular motion," IET Radar, Sonar and Navigation, vol. 1, no. 4, pp. 251-260, 2007.

[15] J. M. Hu, W. Zhou, Y. W. Fu, X. Li, and N. Jing, "Uniform rotational motion compensation for ISAR based on phase cancellation," IEEE Geoscience and Remote Sensing Letters, vol. 8, no. 4, pp. 636-640, 2011.

[16] C.-M. Yeh, J. Xu, Y.-N. Peng, X.-G. Xia, and X.-T. Wang, "Rotational motion estimation for ISAR via triangle pose difference on two range-Doppler images," IET Radar, Sonar and Navigation, vol. 4, no. 4, pp. 528-536, 2010.

[17] C.-M. Yeh, J. Xu, Y.-N. Peng, and X.-T. Wang, "Cross-range scaling for ISAR based on image rotation correlation," IEEE Geoscience and Remote Sensing Letters, vol. 6, no. 3, pp. 597-601, 2009.

[18] S.-H. Park, H.-T. Kim, and K.-T. Kim, "Cross-range scaling algorithm for ISAR images using 2-D fourier transform and polar mapping," IEEE Transactions on Geoscience and Remote Sensing, vol. 49, no. 2, pp. 868-877, 2011.

[19] M. D. Xing, R. B. Wu, J. Q. Lan, and Z. Bao, "Migration through resolution cell compensation in ISAR imaging," IEEE Geoscience and Remote Sensing Letters, vol. 1, no. 2, pp. 141-144, 2004.

[20] L. Lucchese, S. Leorin, and G. M. Cortelazzo, "Estimation of two-dimensional affine transformations through polar curve matching and its application to image mosaicking and remotesensing data registration," IEEE Transactions on Image Processing, vol. 15, no. 10, pp. 3008-3019, 2006.

[21] J. A. Nelder and R. Mead, "A simplex method for function minimization," The Computer Journal, vol. 7, no. 4, pp. 308-313, 1965.

[22] L. I. Xi, G. Liu, and J. Ni, "Autofocusing of ISAR images based on entropy minimization," IEEE Transactions on Aerospace and Electronic Systems, vol. 35, no. 4, pp. 1240-1252, 1999.

[23] B. Zhou, Y. Peng, C. M. Yeh, and J. Tang, "GPGPU accelerated fast convolution back-projection for radar image reconstruction," Tsinghua Science and Technology, vol. 16, no. 3, pp. 256263, 2011. 


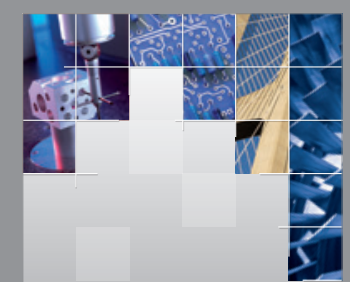

\section{Enfincering}
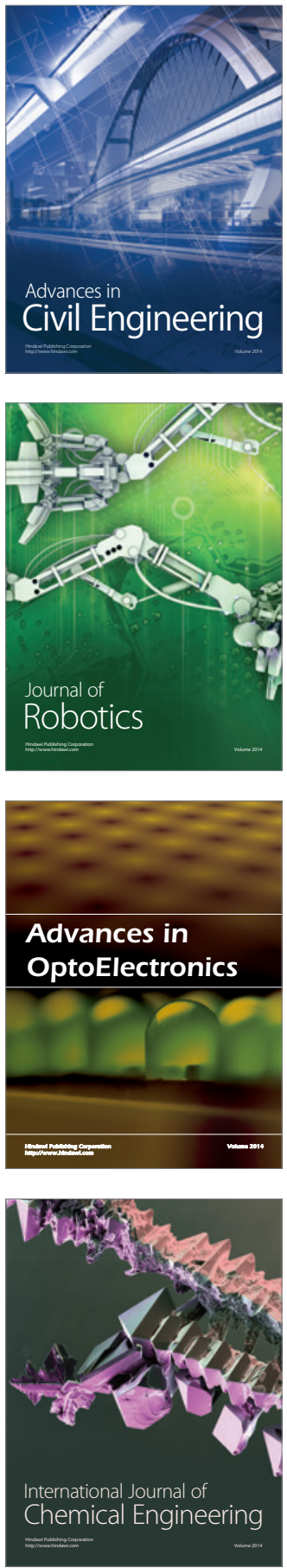

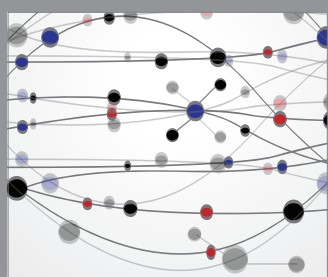

The Scientific World Journal

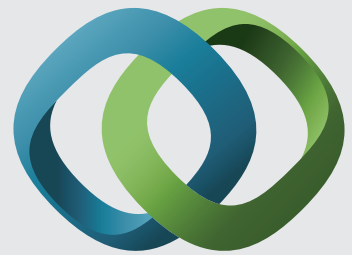

\section{Hindawi}

Submit your manuscripts at

http://www.hindawi.com
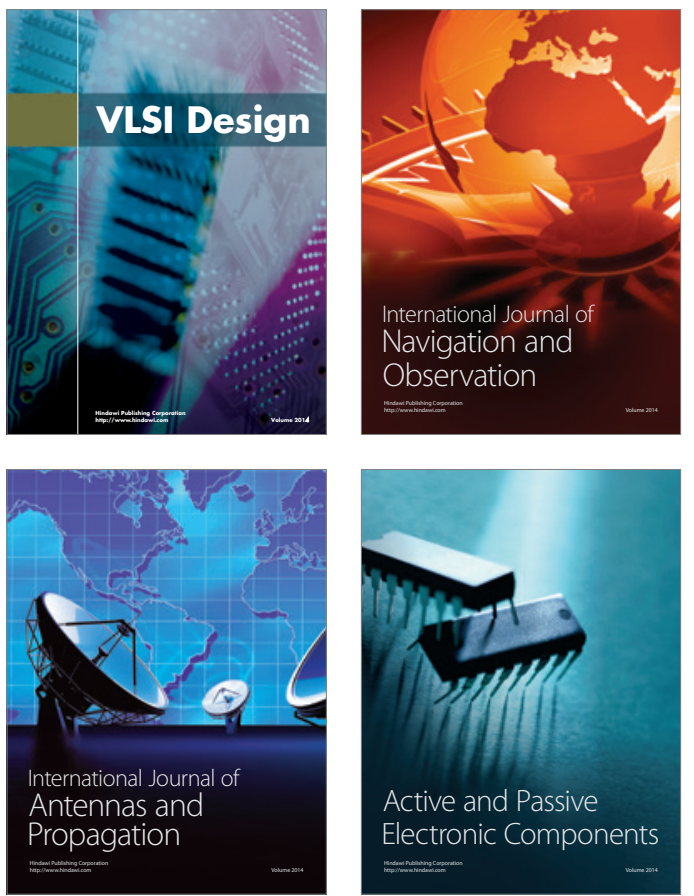
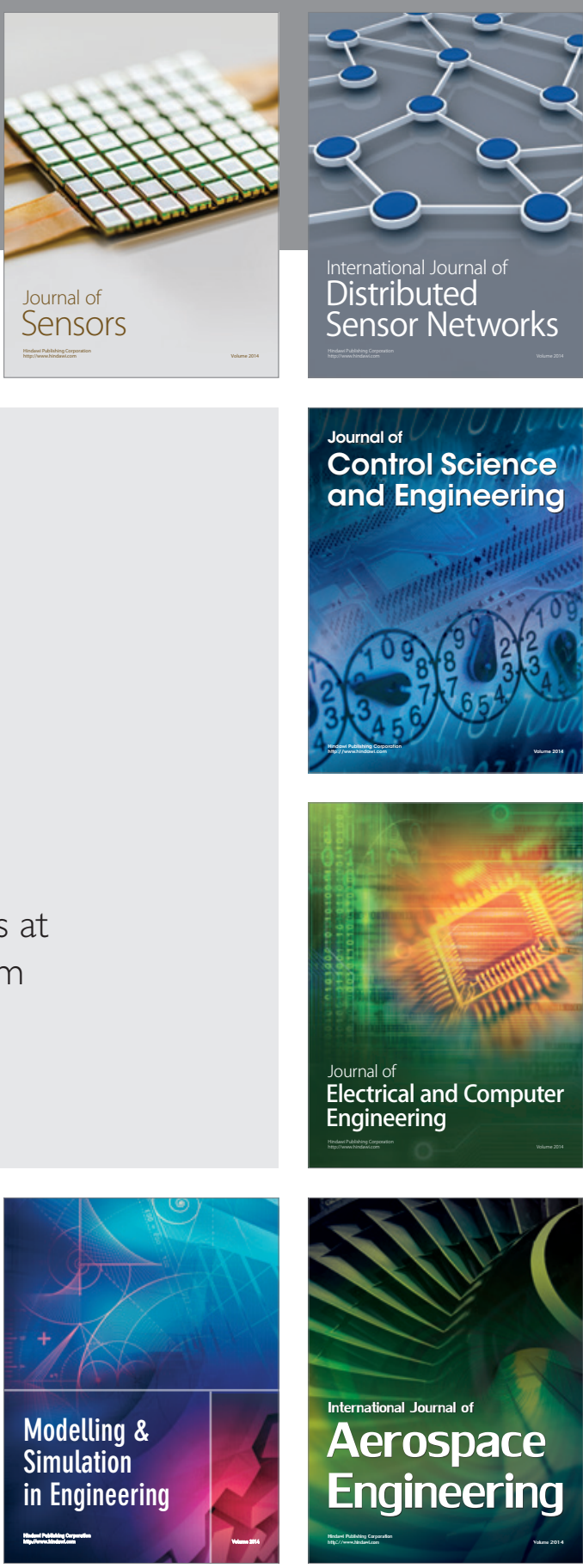

International Journal of

Distributed

Sensor Networks

Journal of

Control Science

and Engineering
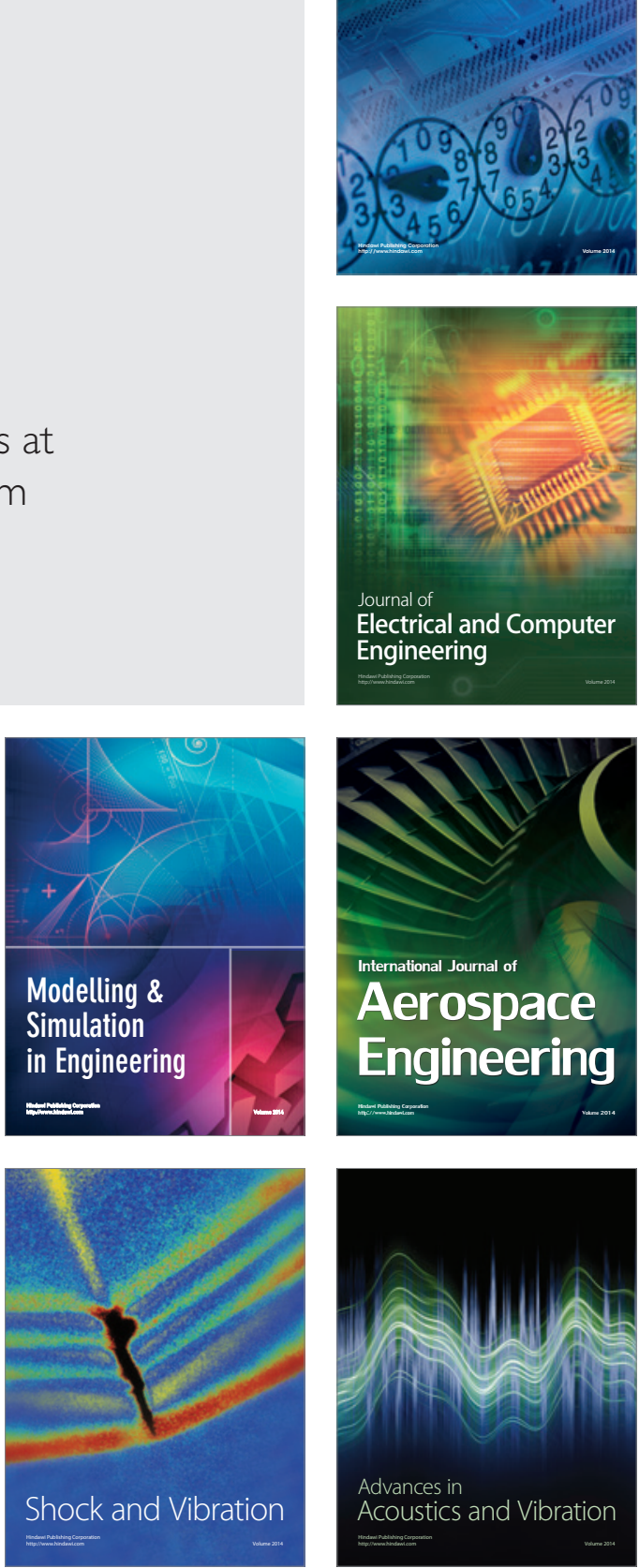